\title{
CT Based Analysis of Arthroscopic Femoral Tunnel Placement in Single Bundle Primary ACL Reconstruction Using Medial Portal Technique
}

\author{
Ahmed Abdul Ghaffar ${ }^{1}$, Atul Agrawal ${ }^{2}$, Rajesh Maheshwari ${ }^{3}$, \\ Shailender Raghuvanshi ${ }^{4}$ \\ ${ }^{I}$ Department Of Orthopaedics, Himalayan Institute Of Medical Sciences, SRH University, Swami Ram Nagar, \\ Dehradun, Uttarakhand, India. \\ ${ }^{2}$ Associate Professor, Corresponding Author, Department Of Orthopaedics, Himalayan Institute Of Medical \\ Sciences, SRH University, Swami Ram Nagar, Dehradun, Uttarakhand, India. \\ ${ }^{3}$ Professor \& Head, Department Of Orthopaedics, Himalayan Institute Of Medical Sciences, SRH University, \\ Swami Ram Nagar, Dehradun, Uttarakhand, India. \\ ${ }^{4}$ Professor \& Head, Department Of Radiodiagnosis, Himalayan, Institute Of Medical Sciences, SRH University \\ Swami Ram Nagar, Dehradun, Uttarakhand, India.
}

\begin{abstract}
:
Background: Achieving anatomical graft placement remains one of the major challenges in ACL reconstruction. In this study we analyzed the anatomic femoral tunnel placement of single bundle endoscopic $A C L$ reconstruction by the medial portal technique using $3 D$ CT reconstruction.

Materials \& Methods: This cross - sectional observational study was performed on 35 consecutive cases of ACL injury presenting over a period of one year. Post-operatively analysis of femoral tunnel placement was done using Mochizuki's method. The data recorded was subjected to standard statistical analysis.

Results: Our study had 29 males and 6 females between 20-50 years of age. In accord with the variance from anatomical position of femoral tunnel by Mochizuki's method expressed as percentage $\{38.7 \% \pm 2.7 \%$ as reported by Mochizuki et al\}, 18(52\%) patients were within the percentage group of 35-39.9. Between the percentage group of 40-44.9 there were 10(29\%) patients while 4(11\%) were between the percentage group of 30-34.9 and only 3(8\%) patients were between 45-50.

Conclusions: The use of medial portal technique allows anatomical ACL tunnel placement. This technique is not limited by the choice of graft or fixation and offers the advantage of true parallel screw placement through the same portal as that used for tunnel drilling in the case of interference fixation. The anteromedial portal technique helps in accurate position of the native footprint in ACL reconstruction.
\end{abstract}

Keywords: Femoral tunnel, Anatomical single bundle ACL reconstruction, Medial portal, Mochizuki's method of CT analysis.

\section{Introduction}

Anatomical graft placement is one of the major challenges in Anterior cruciate ligament reconstruction. Anatomic ACL reconstruction improves joint laxity and prevents degeneration of cartilage (2). In ACL reconstruction nowadays, the importance is and should rightly be given to anatomical graft placements to recreate normal physiologic graft tension and more knee kinematics (3).

The medial portal technique facilitates us with more flexibly and accurately placing the graft at native ACL insertion site. Using Three-Dimensional Computed-tomography reconstruction this study analyzes the accuracy of anatomical ACL footprint in femoral tunnel placement by medial portal technique in single bundle primary ACL reconstruction.

Materials and Methods: This study was conducted over a period of 12 months. Subjects were recruited from the Department of Orthopaedics outdoor as well as indoor, after taking written and informed consent. Patients were selected based on our inclusion criteria of single cruciate ligament, closed injury, with no bony fracture around knee, with fused epiphysis and between the age group of 20-50 years. Patients were also excluded from our study if they had an ACL re-injury or those with an associated ipsilateral lower limb fracture around knee and the patients not willing to undergo follow up 3D - CT scan because of X-ray exposure. The patients were then worked-up for Arthroscopic ACL reconstruction. Routine pre-operative preparations were done.

Surgical technique- Intra-operatively using Arthroscope, diagnostic evaluation of the knee was done and ACL injury was confirmed. Autologous graft of Gracilis and Semitendinosus was prepared from the same side. Tibial tunnel was prepared using the standard tibial zig. Femoral tunnel was prepared using medial portal 
technique with knee in $120^{\circ}$ of flexion using inside-out technique. In this technique, optimal position for the accessory portal was located at a site (as described in Figure 1) that was as medial as possible but without the risk of damaging the medial femoral condyle by a reamer and as low as possible while avoiding the anterior horn of the medial meniscus.

Using the lateral intercondylar ridge as an anatomical landmark, taking care not to cause damage to the remnant fibers of the ACL on the femur, a mark was made at the center of the femoral insertion of the ACL with a free-hand technique using a microfracture awl and a guide wire was inserted through the low anteromedial portal (as shown in Figure 2).

With the knee in full flexion, the tunnel was drilled using a reamer with the same diameter of the graft (4). Graft placement and anchoring was done using Endobutton on the femoral site and Interference screw on the tibial site. Ligament laxity was checked under Arthroscopic view and a stable construct with no laxity was achieved.

Post- operative- A knee ROM brace was applied at the operated knee to counter valgus/varus forces. 3D Computed Tomography analysis of the femoral tunnel was done to assess the anatomic location of the ACL reconstruction (Figure 3).

Methods- Based on the CT- Analysis, a pre designed patient proforma was filled-up. The data recorded was subjected to standard statistical analysis. Quantitative data was expressed in terms of mean \pm standard deviation. Qualitative data was expressed in terms of frequency or percentage.

The CT-based analysis was done by using the Mochizuki's method. A line passing through the center of the femoral tunnel in parallel with the Blumensaat's line was drawn between the deep and shallow subchondral margins of the lateral condyle (as shown in Figure 4).

The distance from the tunnel center to the deep margin was defined as A1 and to the shallow margin as A2 (5). The ratio of A1 to A1+A2 was expressed as percentage $\{38.7 \% \pm 2.7 \%$ as reported by Mochizuki et al $\}$. The results obtained were subjected to standard statistical analysis.

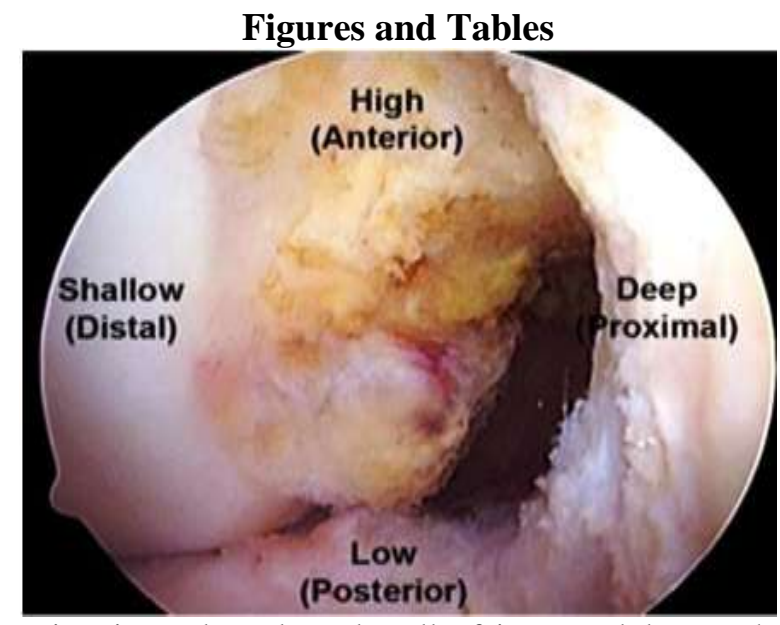

Figure 1: AM portal view. Directions along lateral wall of intercondylar notch- high/superior, low/inferior, shallow and deep. Directions using the anatomical terminology- anterior, posterior and distal. Remnant fibres of native ACL are seen along lower third of lateral wall of the notch.
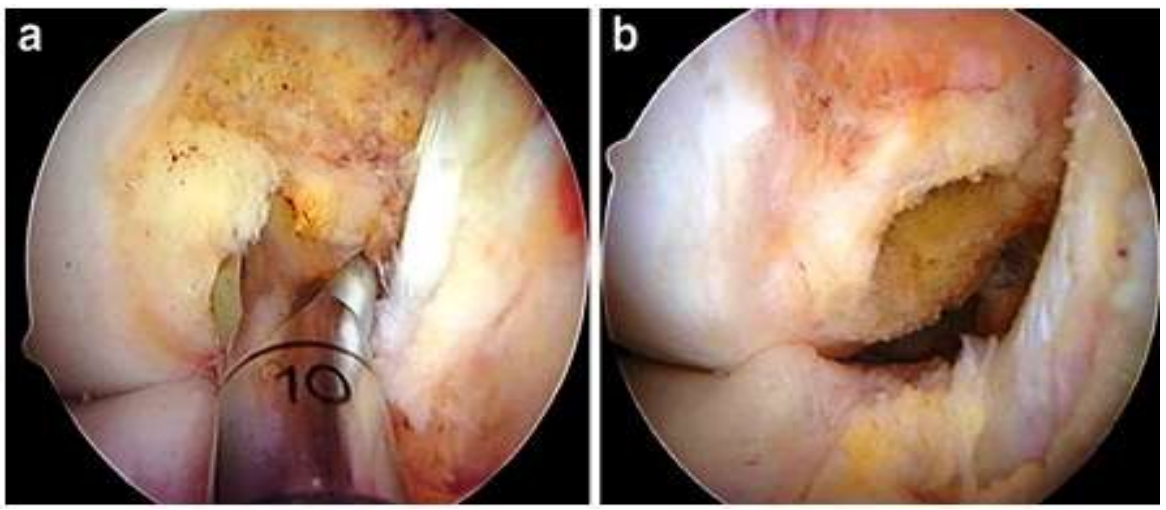

Figure 2: 
a) AM portal view in hyperflexion. Drill bit is inserted through AM portal and lies at centre of ACL femoral attachment site.

b) AM portal view at $90^{\circ}$. Elliptically shaped aperture of ACL femoral tunnel is centred in ACL footprint.

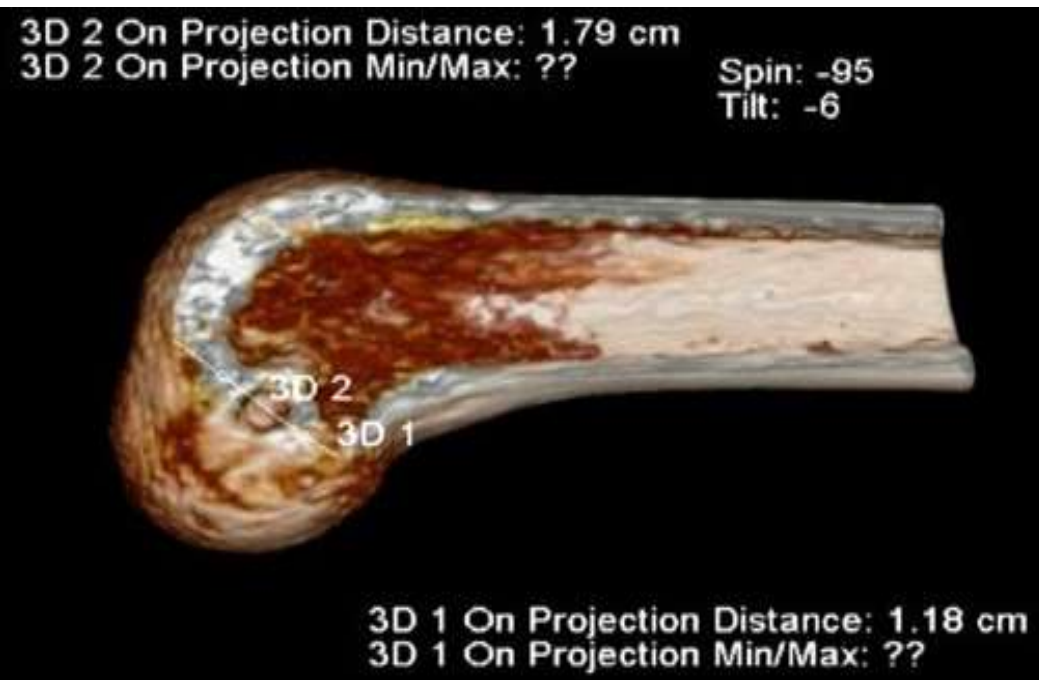

Figure 3: 3D CT Reconstruction by Mochizuki’s method.

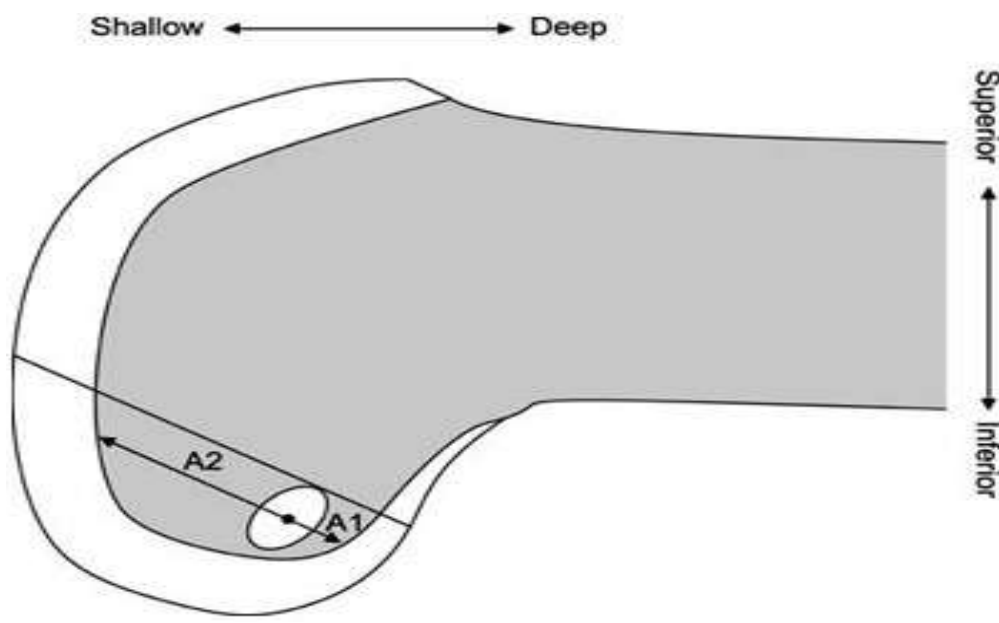

Figure 4: Illustration of Mochizuki's method. The central point of femoral tunnel was calculated as $\mathrm{A} 1 /(\mathrm{A} 1+\mathrm{A} 2)$. A1: the distance between deep border and central point of the tunnel along Blumensaat's line, A2: the distance between the shallow border and central point.

\begin{tabular}{|c|c|c|}
\hline Ligaments & Findings & Percentage \\
\hline ACL- tear & 29 & $83 \%$ \\
\hline ACL- lax & 4 & $11 \%$ \\
\hline ACL- avulsed & 2 & $6 \%$ \\
\hline PCL- tear & 0 & $0 \%$ \\
\hline PCL- lax & 0 & $0 \%$ \\
\hline Medial meniscus- tear & 18 & $52 \%$ \\
\hline Lateral meniscus- tear & 8 & $23 \%$ \\
\hline
\end{tabular}

Table 1: Distribution of patients according to the intra-operative findings of the condition of different ligaments in diagnostic arthroscopy $(n=35)$. 


\begin{tabular}{|c|c|}
\hline $\begin{array}{c}\text { Range of position expressed in \% } \\
\text { (as per Mochizuki's method) }\end{array}$ & Number of patients \\
\hline $30-34.9$ & $4(11 \%)$ \\
\hline $35-39.9$ & $18(52 \%)$ \\
\hline $40-44.9$ & $10(29 \%)$ \\
\hline $45-50$ & $3(8 \%)$ \\
\hline
\end{tabular}

Table 2: Variance from anatomical position of Femoral tunnelaccording to Mochizuki's method ( $\mathrm{n}=35)$.

\section{Conclusion}

Results: The patients were selected between 20-50 years age group. Maximum number of patients were found to be between 21-30 years. 29 males and 6 females sustained the injury in our study. The surgery was performed on the Right knee in 26 and left knee in 9 patients.

In our study, on clinical examination pre-operatively, the Anterior drawer test was positive in all 35 patients, Posterior drawer test was not positive in any of the patients (as per our criteria of single cruciate ligament injury) while, Mcmurray's test was positive in 21 patients for Medial meniscus and positive in 5 patients for Lateral meniscus. Intra-operatively it was found that ACL was torn in 29 of the patients and lax in 4 of them and avulsed in 2 patients, while PCL was torn/lax in none. Medial meniscus was found to be torn in 18 of the patients and lateral meniscus in 8 of them (Table 1).

The CT-based analysis was done by using the Mochizuki's method as described earlier (5). In our study maximum number of patients ie. 18 (52\%) were found to be within the percentage group of 35-39.9. Between the percentage group of 40-44.9 there were $10(29 \%)$ patients while there were $4(11 \%)$ patients between the percentage group of 30-34.9. There were only $3(8 \%)$ patients found to be between the percentage group of 45-50 (Table 2).

\section{Discussion}

Rupture of the anterior cruciate ligament (ACL) is one of the most common knee injuries. Due to the unsatisfactory outcomes of conservative treatment for ACL injuries, reconstruction surgery remains the treatment of choice in most young patients who want to maintain an active lifestyle. The main aims of ACL reconstruction are to restore intact knee stability and normal knee kinematics after reconstruction (6).

Achieving anatomical graft placement remains one of the major challenges in Anterior cruciate ligament reconstruction. The inability of ACL reconstruction to achieve normal knee motion has been thought to be an important factor contributing to the associated joint degeneration after ACL reconstruction. Anatomically placed grafts are believed to more closely reproduce native ACL function and knee kinematics. However, recent studies have suggested that non-anatomic placement of the graft might be a frequent problem during ACL reconstruction (1). Anatomic anterior cruciate ligament (ACL) reconstruction is emphasized to improve joint laxity and to potentially avert initiation of cartilage degeneration. There is a paucity of information on the efficacy of ACL reconstructions by currently practiced tunnel creation techniques in restoring normal joint laxity (2).

The kinematics of the reconstructed knee can be affected by the location of the tibial and femoral tunnels. Recommendations for tunnel placement have changed, and emphasis is laid on anatomical graft placements to recreate normal physiologic graft tension and more knee kinematics (3).

Characterization of the insertion site anatomy in anterior cruciate ligament reconstruction has recently received increased attention in the literature, coinciding with a growing interest in anatomic reconstruction (7). For achieving the most anatomic position for the graft requires the femoral and tibial tunnels to be drilled independently. Use of the anteromedial portal technique provides us with more flexibility in accurately placing the femoral tunnel in the true ACL insertion site. This technique is not limited by the choice of graft or fixation and offers the advantage of true parallel screw placement through the same portal as that used for tunnel drilling in the case of interference fixation (8). Recent studies by Abebe et al, Forsythe et al have validated the use of 3D CT scans and MRI for evaluation of ACL bone tunnel placement postoperatively. The authors have questioned the reliability of conventional radiographs to evaluate ACL bone tunnel placement (Forsythe et al). MRI is a good imaging modality for direct visualization of the ACL graft as stated by McCauley et al. in 2003 and Moon et al. in 2008. However, there have been no studies on the reliability of MRI scans to document bone tunnel placement following ACL reconstruction. Recently, new 3D viewing and measurement methods have developed for visualization of the ACL reconstruction. These methods use CT data and an immersive virtual reality system (9).

We undertook a study of 35 patients for CT based evaluation of anatomical tunnel placement of ACL reconstruction using the medial portal technique. The significance of our study is that it is one of the few studies on ACL reconstruction using a free-hand technique with an accessory medial portal and the evaluation of the postoperative tunnel position and obliquity, with accuracy of CT (3D) based measurements. 
In our study we observed that most of the patients who sustained the injury were young adult males, primarily those associated with different kinds of sports activities which further emphasizes the fact that the injured ACL ought to be treated rapidly in the active young adults to prevent the complication of early joint degeneration and eventually secondary osteoarthritis of the affected knee. One particular finding that we came across during our course of this study was that medial meniscus was more commonly injured in conjunction with the ACL injury as compared to the lateral meniscus but if we go according to the literature, ACL injury is mostly associated with lateral meniscus tear, a point which we found difficult to explain. One advantage which we had experienced intra-operatively by using the medial portal technique for ACL reconstruction was that it places the surgeon at ease when we correlate it with the transtibial technique which requires the patient's knee to be arched at a right angle and puts the surgeon in a strenuous position and we had also perceived that the medial portal technique requires less of assistance during the course of surgery. One difficulty which we faced amidst the surgery was that during the time of labeling at the femoral site of the tunnel at the ACL footprint an appreciable amount of eyeballing is required and we personally felt that there is a vital need for some study to more specifically locate the site for the advantage of the surgeon. Some other problems which we experienced were that after passing the graft from the tibial side along the tunnel, there is an abundance of strength and skill required to pull out the graft at the femoral site which needs to be more simplified and the false impression of the flipping of the endobutton without the use of a radiographic image guidance.

Mochizuki et al performed a study on observation of cadaveric knee for describing anatomic femoral tunnel placement for two-bundle anterior cruciate ligament reconstruction and had concluded that the center of the tunnel was located at $38.7 \% \pm 2.7 \%$ from the deep subchondral margin. These findings were directly coherent with our study in which $52 \%$ of the patients were found to be within the percentage group of 35-39.9 and $29 \%$ of the patients were found to be within the percentage group of 40-44.9. Most of the authors who have performed studies similar to ours, in the past have gone for collateral methods of evaluation of the femoral tunnel placement. We in our study had opted for the Mochizuki's method as we felt that emphasis must be laid on different, useful and almost virgin methods of evaluation of the anatomic tunnel placement in ACL reconstruction to further prove our point.

In this study we concluded that the use of medial portal technique in which the ACL femoral tunnel is drilled through an anteromedial or accessory anteromedial portal allows consistent anatomical ACL femoral tunnel placement. Use of the anteromedial portal technique provides us with more flexibility in accurately placing the femoral tunnel in the true ACL insertion site. This technique is not limited by the choice of graft or fixation and offers the advantage of true parallel screw placement through the same portal as that used for tunnel drilling in the case of interference fixation. In anatomic single bundle ACL reconstruction, the anteromedial portal technique can restore accurate position of the native footprint.

Though outside the domain of this study, but we still sincerely suggest that if the ACL reconstruction is at the anatomical footprint the post-operative rehabilitation and stability of the knee improves significantly, a fact that would doubtlessly help in gaining better functional results and that too in decreased time period.

The limitations of this study are that the sample size is not very big and we have not been able to include functional results due to the longer follow up needed for it. In future we plan to do a comparison of 3D CT based accuracy of femoral tunnel with the functional results of these ACL reconstructions.

\section{References}

[1]. Chong Bum Chang,Ja-Young Choi, In Jun Koh, Kil Jae Lee,Kyung-Hag Lee, Tae Kyun Kim. Comparisons of Femoral Tunnel Position and Length in Anterior Cruciate Ligament Reconstruction:ModifiedTranstibial Versus Anteromedial Portal Techniques.Arthroscopy. 2011;27(10):1389-94.

[2]. Sim JA, Gadikota HR, Li JS, Li G, Gill TJ. Biomechanical evaluation of knee joint laxities and graft forces after anterior cruciate ligament reconstruction by anteromedial portal, outside-in, and transtibial techniques.Am J Sports Med. 2011;39(12):2604-10.

[3]. Hong-Chul Li, Yong-Cheol Yoon, Joon-Ho Wong, Ji-Hoon Bae. Anatomical versus non-anatomical single bundle anterior cruciate ligament reconstruction: a cadaveric study of comparison of knee stability. ClinOrthop Surg. 2012;4(4):249-55.

[4]. Kwang W L, Yoon S H, Yong J C, Yang D S, Kim H Y, Won S C.Anatomic Single Bundle Anterior Cruciate Ligament Reconstruction by Low Accessory Anteromedial Portal Technique: An In Vivo 3D CT Study.Knee Surgery \& Related Research. 2014;26(2):97-105.

[5]. Mochizuki T, Muneta T, Nagase T, Shirasawa S, Akita KI, Sekiya I. Cadaveric knee observation study for describing anatomic femoral tunnel placement for two-bundle anterior cruciate ligament reconstruction. Arthroscopy. 2006;22:356-61.

[6]. Kim H S, Jong K S, Ah Reum Jo. Current Trends in Anterior Cruciate Ligament Reconstruction. Knee Surgery \& Related Research. 2013;25(4):165-73.

[7]. Forsythe B, Kopf S, Wong AK, Martins CA, Anderst W, Tashman S, et al. Location of femoral and tibial tunnels in anatomic double-bundle anterior cruciate ligament reconstruction analyzed by three-dimensional computed tomography models. J Bone Joint Surg Am. 2010;92(6):1418-26.

[8]. Christopher D. Harner, Nicholas J. Honkamp, Anil S. Ranawat. Anteromedial Portal Technique for Creating the Anterior Cruciate Ligament Femoral Tunnel. Arthroscopy. 2008;24(1):113-5.

[9]. Duncan E Meuffels, Jan-Willem, Anton H J Koning, Charles H Brown Jr, Jan A N Verhaar, Reijman M. Visualization of postoperative anterior cruciate ligament reconstruction bone tunnels. Acta Orthopaedica. 2011;82(6):699-703. 